$R M x A C, \mathbf{5 2}, 23-24(2020)$

(C) 2020: Instituto de Astronomía, Universidad Nacional Autónoma de México

https://doi.org/10.22201/ia.14052059p.2020.52.09

\title{
RED Be CANDIDATE STARS IN THE MAGELLANIC CLOUDS
}

\author{
K. Vieira ${ }^{1}$, A. García-Varela ${ }^{2}$, and B. Sabogal ${ }^{2}$
}

RESUMEN

En este trabajo revisitamos el tema de las estrellas candidatas Be hacia las Nubes de Magallanes, previamente estudiado por los autores usando movimientos propios del catálogo SPM4. Obtuvimos paralajes y movimientos propios de GAIA DR2 para 2357 y 994 LMC y SMC candidatas Be, respectivamente. Las paralajes y movimientos propios vs el color $V-I$ fácilmente revelán la presencia de la contaminacion producida por estrellas rojas que pertenecen a la Vía Láctea, confirmando los resultados obtenidos en nuestro trabajo anterior sobre este tema. Pero esta vez, encontramos unas pocas candidatas Be rojas que se comportan claramente como miembros de las Nubes de Magallanes. Se hizo una evaluación de membresía a las Nubes de Magallanes para cada candidata Be, en base a la distribución de sus paralajes y movimientos propios. Magnitudes IRSF $J H K s$ se obtuvieron para aproximadamente el $70 \%$ de las candidatas Be con datos astrometricos en GAIA DR2. Varias de las candidatas Be tienen colores ópticos e infrarrojos significativamente mas rojos que los esperados para estrellas Clásicas Be.

\section{ABSTRACT}

We revisit the subject of Be star candidates towards the Magellanic Clouds, previously studied by the authors using SPM4 proper motions. We obtain GAIA DR2 parallaxes and proper motions for 2357 and 994 LMC and SMC Be candidates, respectively. Parallaxes and proper motions vs. color $V-I$ easily reveal the presence of the redder galactic contaminant foreground, as concluded in our previous work, but this time we do find a few red Be stars candidates consistent with being true Magellanic objects. A membership assessment to each Magellanic Cloud is done for each Be candidate, based on the distribution of their parallaxes and proper motions. Near-infrared IRSF JHKs magnitudes were obtained for about $70 \%$ the Be stars candidates with GAIA DR2 astrometric data. Several Magellanic Be candidates are redder in optical an near-infrared than what is expected for Classical Be stars.

Key Words: proper motions — stars: emission-line, Be

\section{INTRODUCTION}

Be stars are broadly defined as non-supergiant (luminosity class II to V) B-type stars that have or have had Balmer emission lines. The presence of a flattened circumstellar gaseous disk formed of material ejected from the star, a dust-free Keplerian decretion disc, is currently the accepted explanation for some of the observed features in Be stars (see Rivinius et al. (2013) for details). In the so-called Classical $\mathrm{Be}(\mathrm{CBe})$ the disk comes from the rapid rotation of the star. In this investigation, the results in Vieira et al. (2017), are tested with recently released GAIA DR2 (GAIA Collaboration et al. 2018), that has significantly better proper motions and also measured parallaxes that were not available before. The main result regarding the redder candidates be-

\footnotetext{
${ }^{1}$ Centro de Investigaciones de Astronomía, Apartado Postal 264, Mérida 5101. Mérida, Venezuela (kvieira@cida.gob.ve).

${ }^{2}$ Universidad de los Andes, Departamento de Física, Carrera 1 No 18A-10, Bloque Ip, A.A. 4976, Bogotá, Colombia (josegarc@uniandes.edu.co, bsabogal@uniandes.edu.co).
}

ing contaminants was indeed confirmed, but several red Be candidates emerged as true Magellanic stars. Though most of them are classified as Type-4 (see Mennickent et al. (2002) for this classification, according to their variability light curve morphology), and are considered by them and other references thereafter as surely CBe stars, they do not occupy the infrared vs optical colours known so far for CBe stars.

\section{GAIA DR2 DATA AND MEMBERSHIP TO THE MAGELLANIC CLOUDS}

Be star candidates for the LMC and SMC were obtained from Sabogal et al. (2005) and Mennickent et al. (2002), respectively, where a total of 2446 and 1019 candidates are listed. After eliminating repeated entries with very similar photometry $(<0.1$ magnitude differences) in the three $B V I$ bands, the clean lists of Be candidates have 2393 and 1004 stars, towards the LMC and SMC, respectively. The GAIA DR2 match was chosen as the star having the lowest 


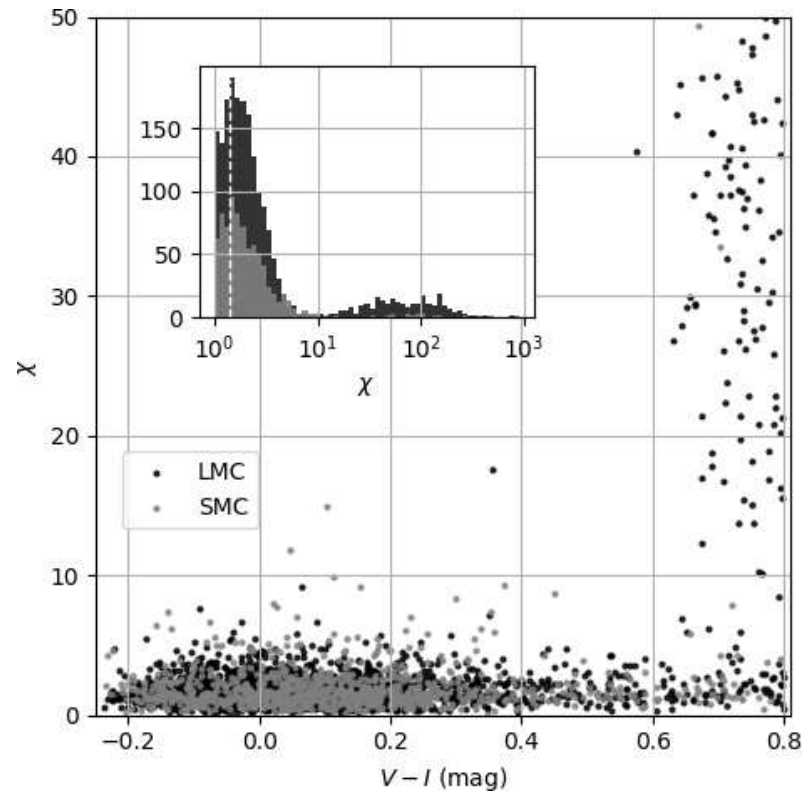

Fig. 1. Plot of $\chi$ values vs $V-I$ for the LMC (black) and SMC (grey) Be candidates. Inset figure shows the histogram in a log-scale of $\chi$ values, that encompasses all Be candidates. Vertical white dashed marks $\chi=\sqrt{2}$.

value of

$$
\text { posmag_ranking }=\sqrt{\left(\frac{\Delta \theta}{\theta_{\max }}\right)^{2}+(V-G)^{2}}
$$

where $\Delta \theta$ is the sky angular separation in arcseconds between the (RA,DEC) coordinates listed for the Be candidates and the GAIA DR2 ones, $\theta_{\max }=2^{\prime \prime}$ for the SMC and $\theta_{\max }=4^{\prime \prime}$ for the LMC. Based on a sample of best measured blue stars, with GAIA DR2 data visibly clustered around the values expected for the Clouds, we found the mean values for parallax and each proper motion coordinate, and then evaluated the normalized data given by:

$$
\text { datum }_{\text {norm }}=\frac{\text { datum }- \text { population mean }}{\text { datumerror }} .
$$

Each of them followed a normal distribution around zero but with dispersions visibly larger than 1, revealing that original quoted errors were underestimated. We found a scaling value that converted these distributions into a $N(0,1)$ and with such computed standardized values for all Be candidates. Magellanic stars cluster around zero simultaneously in standardized parallax and proper motions. This leads us to define a new variable $\chi$, as follows:

$$
\chi=\sqrt{\left(\varpi_{s t d}\right)^{2}+\left(\mu_{\alpha} \cos (\delta) s t d\right)^{2}+\left(\mu_{\delta} s t d\right)^{2}}
$$

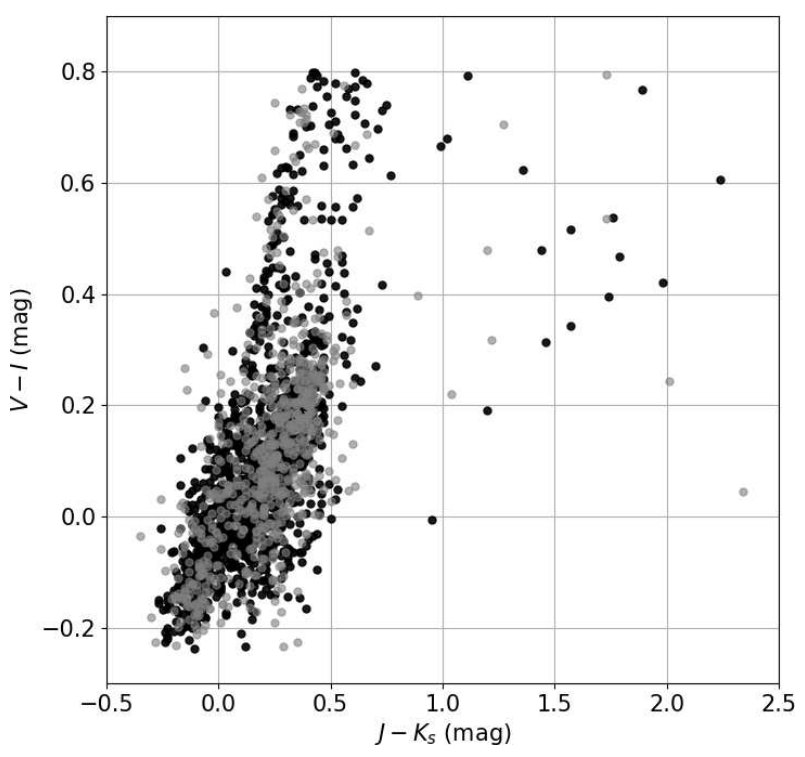

Fig. 2. Color-color diagram for LMC (black) and SMC (grey) single population final samples stars.

which follows closely a $\chi$ (Chi) distribution with 3 degrees of freedom. Such probability distribution has a mode at $\chi=\sqrt{2}$, and $\chi \leq 3.389$ should enclose enclose $99 \%$ of the Magellanic population, if all standardized data truly followed $N(0,1)$ distributions and were also free of contamination. Figure 1 plots $\chi$ vs $V-I$ for the LMC and SMC data, and from the distribution of both the bluer Magellanic population and the redder Galactic one, we decide to draw a limit between the two at $\chi=10$. We believe that incomplete standardization of the most extreme data within the Magellanic populations has caused them to stray beyond the expected values for the $\chi$ distribution. In any case. our standardized data are good in a relative sense, since Galactic foreground stars distribute themselves visibly far away from the cluster of Magellanic data points around zero. Our final single Magellanic population samples, with all stars with $\chi<10$, yield 2109 stars in the LMC and 974 stars in the SMC, respectively. Figure 2 shows the $V-I$ vs. $J-K_{s}$ color-color diagram for those samples, in which a number of Be candidates clearly have redder colors than the general concentration occupied by most Be candidates, which corresponds to the locus of CBe stars.

\section{REFERENCES}

GAIA Collaboration, et al. 2018a, A\&A, 616, A1

Mennickent, R. E., et al. 2002, A\&A, 393, 887

Sabogal, B. E., et al. 2005, MNRAS, 361, 1055

Rivinius, T., et al. 2013, A\&ARv, 21, 69

Vieira, K., et al. 2017, MNRAS, 469, 4175 